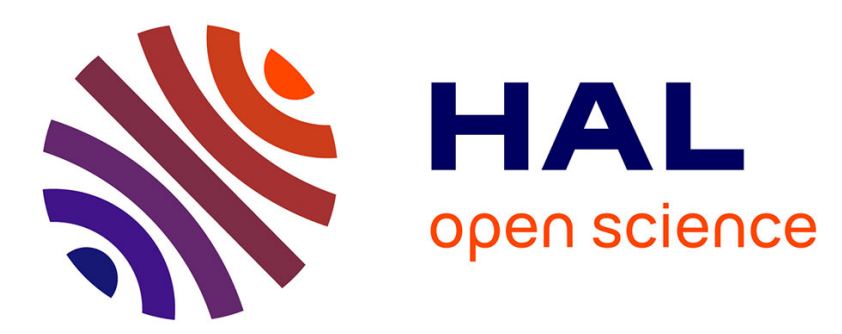

\title{
Frequency dependence of the conductivity in presence of an electric field in one dimension: weak-disorder limit
}

Bernard Derrida, R. Orbach

\section{To cite this version:}

Bernard Derrida, R. Orbach. Frequency dependence of the conductivity in presence of an electric field in one dimension: weak-disorder limit. Physical Review B, 1983, 27 (8), pp.4694-4701. 10.1103/PhysRevB.27.4694 . hal-03285931

\section{HAL Id: hal-03285931 \\ https://hal.science/hal-03285931}

Submitted on 19 Jul 2021

HAL is a multi-disciplinary open access archive for the deposit and dissemination of scientific research documents, whether they are published or not. The documents may come from teaching and research institutions in France or abroad, or from public or private research centers.
L'archive ouverte pluridisciplinaire HAL, est destinée au dépôt et à la diffusion de documents scientifiques de niveau recherche, publiés ou non, émanant des établissements d'enseignement et de recherche français ou étrangers, des laboratoires publics ou privés. 


\title{
Frequency dependence of the conductivity in presence of an electric field in one dimension: Weak-disorder limit
}

\author{
B. Derrida \\ Service de Physique Théorique, Centre d'Etudes Nucléaires de Saclay, \\ 91191 Gif-sur-Yvette Cedex, France \\ R. Orbach* \\ Ecole Supérieure de Physique et de Chimie industrielles de Paris 10 rue Vauquelin, 75005 Paris, France
}

(Received 20 September 1982)

\begin{abstract}
The frequency dependence of the electrical conductivity is examined for a classical hopping model of a random one-dimensional system in the presence of a superposed static electric field. The effect of the field is taken as a constant bias for the left-right jump rates. A general expression is derived for the mean velocity and frequency-dependent conductivity. Explicit evaluation of these equations is given for correlated and uncorrelated hopping rates: (1) in general for high frequencies and (2) to lowest order in the disorder for all frequencies. In the latter case, an initial decrease in $\sigma(\omega)$ for very small $\omega$ is found, $\sigma_{R}=a_{0}-a_{1} \omega^{2}$, $\sigma_{I}=a_{2} \omega^{3}$. For larger frequencies, the conductivity crosses over to the form $\sigma(\omega)=b_{0}+b_{1}(i \omega)^{1 / 2}$, previously calculated by Alexander and Orbach. The $a_{i}, b_{i}$ are constants which depend on the strength of the bias and the randomness. The crossover frequency increases with the bias. In addition, the variance in the autocorrelation function is calculated in the long-time limit, for weak disorder in the symmetric case. It is shown that fluctuations do not significantly affect the determination of this quantity under these conditions.
\end{abstract}

\section{INTRODUCTION}

The frequency dependence of the electrical conductivity in one-dimensional systems with symmetrical random hopping rates has been calculated recently in a series of papers. ${ }^{1-3}$ Recent electrical conductivity experiments on the quasi-onedimensional conductor quinolinium ditetracyanoquinodimethanide $\left[\mathrm{Qn}(\mathrm{TCNQ})_{2}\right]$ have been analyzed using these results by Alexander et $a .^{4}$ All of these studies, with the exception of Ref. 3, have only treated the case of symmetric hopping rates, and hence were only applicable in the small electric field regime.

Recently, attention has been directed to the question of transport with random, but biased, hopping rates. $^{5-7}$ These calculations have explored the time dependence of the mean displacement. Our purpose is to calculate the frequency dependence of the conductivity in the nonlinear electric field regime. We shall find a result very different from the symmetric case at the lowest of frequencies, crossing over to a response which is the same as found for symmetric hopping at higher frequencies. This crossover has been anticipated in Ref. 3.
Our purpose here is to formulate the problem in such a manner that the frequency dependence of the conductivity can be calculated directly. Section II describes the formal calculation of the ac conductivity in a uniform dc field. Section III exhibits the high-frequency limit for the conductivity. The solution for weak disorder is developed in Sec. IV, with figures exhibiting the real and imaginary part of the frequency response for different biases. In both Secs. III and IV we treat the case of correlated and uncorrelated hopping rates. In Sec. V, we derive an expression for the variance of the autocorrelation function in the weak-disorder limit, and show that fluctuations are unimportant in this, and the longtime, limit. Finally, we summarize our findings in Sec. VI.

\section{FORMAL SOLUTION FOR THE ac CONDUCTIVITY IN A UNIFORM dc FIELD}

We treat the same master equation for hopping transfer as in Ref. 1, but with asymmetric hopping rates. Let $P_{n}(t)$ be the probability that site $n$ is occupied at time $t$. Then, for near-neighbor hops only, 


$$
\frac{d P_{n}}{d t}=Y_{n+1} P_{n+1}+X_{n-1} P_{n-1}-\left(X_{n}+Y_{n}\right) P_{n},
$$

where $Y_{n+1}$ is the hopping rate to the left (from $n+1$ to $n)$ and $X_{n-1}$ is the hopping rate to the right (from $n-1$ to $n$ ). In the presence of an applied static electric field $\mathscr{C}_{0}$, and a small oscillating field $\mathscr{E}$ at frequency $\omega$,

$$
\begin{aligned}
Y_{n} & =\left[\exp \left(-\frac{\left(\mathscr{E}_{0}+\mathscr{E} e^{i \omega t}\right) e l}{k_{B} T}\right)\right] W_{n} \\
& \equiv\left[\exp \left(-2 \frac{\left(\mathscr{E}_{0}+\mathscr{E} e^{i \omega t}\right) e l}{k_{B} T}\right)\right] X_{n-1},
\end{aligned}
$$

where we have taken the potential drop to be the same along all segments of equal length $l$, and where $W_{n}$ is the symmetric hop rate between $n-1$ and $n$ (i.e., the hop rate in the absence of an applied static field). Expanding in $\mathscr{E}$,

$$
Y_{n}=a\left(1-E e^{i \omega t}\right) X_{n-1},
$$

where $E=2 \mathscr{C}_{e l} / k_{B} T$ and $a=\exp \left(-2 \mathscr{E}_{0} e l / k_{B} T\right)$ is termed the bias. The velocity of the particle at time $t$ is given by

$$
\begin{aligned}
V & =\frac{d}{d t} \frac{\sum_{n} n P_{n}(t)}{\sum_{n} P_{n}(t)} \\
& =\frac{\sum_{n}\left(X_{n}-Y_{n}\right) P_{n}(t)}{\sum_{n} P_{n}(t)} .
\end{aligned}
$$

Expanding $P_{n}(t)$ in a power series in the reduced field $E$,

$$
P_{n}(t)=Q_{n}+R_{n} E e^{i \omega t}+\cdots,
$$

one can derive expressions for $Q_{n}$ and $R_{n}$ from Eq. (1). One finds

$$
\begin{gathered}
X_{n}\left(a Q_{n+1}-Q_{n}\right)-X_{n-1}\left(a Q_{n}-Q_{n-1}\right)=0, \\
i \omega R_{n}=X_{n}\left(a R_{n+1}-R_{n}\right)-X_{n-1}\left(a R_{n}-R_{n-1}\right) \\
\quad-a X_{n} Q_{n+1}+a X_{n-1} Q_{n} .
\end{gathered}
$$

Once $Q_{n}$ and $R_{n}$ are known, we may find $V$ from Eqs. (5) and (4). From Eq. (6b), $\sum_{n} R_{n}=0$ whence

$$
V=\frac{\sum_{n}\left\{\left[X_{n}-a X_{n-1}\left(1-E e^{i \omega t}\right)\right]\left[Q_{n}+R_{n} E e^{i \omega t}\right]\right\}}{\sum_{n} Q_{n}} \text {. }
$$

Writing the velocity in the form $V=v+E e^{i \omega t} \sigma(\omega)$, one identifies

$$
v=\frac{\sum_{n}\left[\left(X_{n}-a X_{n-1}\right) Q_{n}\right]}{\sum_{n} Q_{n}}
$$

and

$$
\sigma(\omega)=\frac{\sum_{n}\left[\left(X_{n}-a X_{n-1}\right) R_{n}+a X_{n-1} Q_{n}\right]}{\sum_{n} Q_{n}} .
$$

These are our two principal relationships (the first is merely a restatement of the result obtained in Refs. 5-7). We can evaluate Eq. (8) immediately for our model. Expressing the $Q_{n}$ as

$$
Q_{n}=\sum_{p=0}^{\infty} a^{p} / X_{n+p}
$$

Equation (6a) is solved immediately. One finds

$$
v=(1-a) /\left\langle\frac{1}{X_{0}}\right\rangle \text {. }
$$

This is the same result as in Ref. 7 , if we note $a=\exp \left(-2 \mathscr{C}_{0} e l / k_{B} T\right)$ and $\left\langle 1 / X_{0}\right\rangle=\exp \left(\mathscr{E}_{0} e l\right)$ $\left.k_{B} T\right)\langle 1 / W\rangle$, where $W_{n}$ is the symmetric hop rate between $n-1$ and $n$ in the absence of an applied electric field. Inserting into Eq. (11),

$$
v=2\langle 1 / W\rangle^{-1} \sinh \left(\mathscr{E}_{0} e l / k_{B} T\right),
$$

a well-known result, ${ }^{7}$ and one which reproduces Rice et al. ${ }^{8}$ for the case of a regular lattice. We now go on to explicit evaluation of the conductivity, Eq. (9), in the high-frequency (Sec. III) and weakdisorder (Sec. IV) regimes.

\section{HIGH-FREQUENCY LIMIT}

We examine the case when the frequency $\omega$ is much greater than any of the hopping rates $X_{n}, Y_{n}$. Using the relationship Eq. (6b), one expands in inverse powers of $\omega$ to find $R_{n}$ in terms of $Q_{n}$ :

$$
\begin{aligned}
R_{n}=\frac{1}{i \omega} a\left(X_{n-1} Q_{n}-X_{n} Q_{n+1}+\frac{1}{(i \omega)^{2}}\right. & \left(a^{2} X_{n}^{2} Q_{n+1}-a^{2} X_{n} X_{n+1} Q_{n+2}-a X_{n} X_{n-1} Q_{n}+a X_{n}^{2} Q_{n+1}-a^{2} X_{n-1}^{2} Q_{n}\right. \\
& \left.+a^{2} X_{n-1} X_{n} Q_{n+1}+a X_{n-1} X_{n-2} Q_{n-1}-a X_{n-1}^{2} Q_{n}\right)+O\left(\frac{1}{(i \omega)^{3}}\right) .
\end{aligned}
$$


One now uses the expression for $Q_{n}$, Eq. (10), in Eq. (13) to obtain the explicit form for $R_{n}$. Knowing $Q_{n}$ and $R_{n}, \sigma(\omega)$ is known from Eq. (9). We exhibit two forms for $\sigma(\omega)$ : the $X_{n}$ uncorrelated, and the $X_{n}$ correlated. The former becomes

$$
\begin{aligned}
\sigma_{\text {uncor }}(\omega)= & a\langle X\rangle+\frac{1}{i \omega}\left[2 a^{2}\langle X\rangle^{2}-a(a+1)\left\langle X^{2}\right\rangle+a(1-a)\langle X\rangle /\langle 1 / X\rangle\right] \\
& +\frac{1}{(i \omega)^{2}}\left[2 a^{3}\langle X\rangle^{3}-3 a^{2}(1+a)\langle X\rangle\left\langle X^{2}\right\rangle+a(1+a)^{2}\left\langle X^{3}\right\rangle-a(1-a)\left\langle X^{2}\right\rangle /\langle 1 / X\rangle\right]+\cdots .
\end{aligned}
$$

This expansion is, in principle, valid as long as $\omega \gg\left\langle X^{n}\right\rangle^{1 / n}$. This is an interesting form, in that some unusual averages enter for asymmetric transfer rates $(a<1)$. For the symmetric case, $a=1$, and one recovers the highfrequency form for $\sigma(\omega)$ given in Ref. 1 [their Eq. (7.8)]. It is interesting to note that the inverse moment of the $X_{n}$ enters at high frequencies in the asymmetric case. This suggests nonuniversal behavior for distribution of class (c) (in the notation of Ref. 1) even at high frequencies, whereas the behavior is purely universal in the high-frequency regime for symmetric transfer rates.

The correlated case has the following high-frequency conductivity:

$$
\begin{aligned}
\sigma_{\text {cor }}(\omega)= & {[(1-a) /\langle 1 / X\rangle] \sum_{p=1}^{\infty}\left\langle X_{0} / X_{p}\right\rangle a^{p} } \\
& +\frac{1}{i \omega}[(1-a) /\langle 1 / X\rangle]\left[a\langle X\rangle+2 \sum_{p=2}^{\infty}\left\langle X_{0} X_{1} / X_{p}\right\rangle a^{p}-(1+a) \sum_{p=1}^{\infty}\left\langle X_{0}^{2} / X_{p}\right\rangle a^{p}\right)+\cdots,
\end{aligned}
$$

if $\omega \gg\left\langle X^{n}\right\rangle^{1 / n}$. Because of the translational invariance, one can replace averages like $\left\langle X_{0} X_{1} / X_{p}\right\rangle$ by $\left\langle X_{n} X_{n+1} / X_{n+p}\right\rangle$ for any $n$.

In the symmetric limit $(a=1)$ for short-range $(\mathrm{sr})$ correlations, it reduces to

$$
\left.\sigma_{\mathrm{cor}, \mathrm{sr}}(\omega)=\langle X\rangle+\frac{2}{i \omega}\left[\left\langle X_{0} X_{1}\right\rangle-\left\langle X_{0}^{2}\right\rangle\right]+\cdots, \omega\right\rangle\left\langle X^{n}\right\rangle^{1 / n}
$$

\section{WEAK-DISORDER LIMIT}

We pass to the limit of weak disorder in order to obtain a solution of Eq. (6b). To this order the expression for the frequency-dependent conductivity developed below [e.g., Eq. (23)] is exact for all frequencies (see the Summary, Sec. VI, for further discussion of this question). We set

$$
\frac{1}{X_{n}}=A+\epsilon_{n}, \quad\left(\epsilon_{n}^{2}\right)^{1 / 2} \ll A .
$$

In exactly the same manner as before, we can write the exact solution for $Q_{n}$,

$$
Q_{n}=A /(1-a)+\sum_{p=0}^{\infty} a^{p} \epsilon_{n+p}
$$

We must now evaluate $R_{n}$ in terms of the $Q_{n}$ from Eq. (6b). To carry this through (to any order in $\epsilon$, $\sum_{n=-\infty}^{n=\infty} R_{n}=0$ ), we expand,

$$
\begin{aligned}
R_{n}= & \sum_{p=-\infty}^{\infty} \beta_{p} \epsilon_{n+p} \\
& +\sum_{p=-\infty}^{\infty} \sum_{q=-\infty}^{\infty} \gamma_{p, q} \epsilon_{n+p} \epsilon_{n+q}+\cdots,
\end{aligned}
$$

where we shall work only to linear order in $\epsilon$. Using Eqs. (10') and (18) in Eq. (9), one finds to lowest order in the disorder, for uncorrelated $X_{n}$,

$$
\begin{aligned}
\sigma_{\text {uncor }}(\omega)=[ & (1-a) / A] \\
& \times\left\{\left(1 / A^{2}\right)\left(a \beta_{-1}-\beta_{0}\right)\left\langle\epsilon^{2}\right\rangle\right. \\
& +a /(1-a) \\
& \left.+\left[a /(1-a) A^{2}\right]\left\langle\epsilon^{2}\right\rangle\right\},
\end{aligned}
$$

where it remains necessary to calculate the $\beta_{p}$ defined in Eq. (18). The algebra leading to a solution is tedious, and we merely exhibit the results here. For $p \geq 0$.

$$
\begin{aligned}
\beta_{p}= & \frac{\lambda(a-1)}{(\lambda-1)(\lambda-a)} a^{p} \\
& +\frac{(\lambda-a)^{2}}{(1-a)(\lambda-1)\left(\lambda^{2}-a\right)} \mu^{p},
\end{aligned}
$$

and for $p \leq-1$,

$$
\beta_{p}=-\frac{a(\lambda-1)^{2}}{(\lambda-a)(1-a)\left(\lambda^{2}-a\right)} \lambda^{p+1},
$$

where $\lambda$ is the largest root of 


$$
\lambda^{2}-\lambda(1+a+i \omega A)+a=0,
$$

$\mu=a / \lambda$, and $a \leq 1$. In particular, for purposes of Eq. (19),

$$
a \beta_{-1}-\beta_{0}=\frac{a}{1-a} \frac{a+1-2 \lambda}{\lambda^{2}-a},
$$

leading to our principal result,

$$
\sigma_{\text {uncor }}(\omega)=\frac{a}{A}+\frac{\left\langle\epsilon^{2}\right\rangle}{A^{3}} \frac{a(\lambda-1)^{2}}{\lambda^{2}-a} .
$$

The frequency limits of Eq. (23) are interesting, and illuminate the essential physics of the transport process. The behavior of the roots of Eq. (21) are essential to our analysis. If one attempts to make a lowfrequency expansion of $\lambda$, one is faced with a comparison of $(1-a)^{2}$ to $\omega A(1+a)$.

$$
\begin{aligned}
2 \lambda=1 & +a+i \omega A \\
& +\left[(1-a)^{2}+2 i \omega A(1+a)-(\omega A)^{2}\right]^{1 / 2} .
\end{aligned}
$$

Our analysis of this ratio is based on a comparison of the "drift" distance with the "diffusive" displacement, exactly as in Ref. 3 . We shall show that the very small $\omega$ (long-time) limit is dominated by the drift of the particle under the influence of the electric field, the larger $\omega$ domain by diffusion. The former will result in a diminution of $\sigma_{\text {uncor }}(\omega)$ with increasing frequency, the latter in an increase. The former is a consequence of electric field driven drift causing the particle to encounter rare very small transfer rates which diminish the conductivity. It would not have experienced these transfers if its motion were purely diffusive on the same time scale of $1 / \omega$. The latter regime is associated with shorter and shorter root-mean-square distances as $\omega$ increases, leading to fewer encounters with small transfer rates.

The crossover condition can be obtained in exactly the same manner as in Ref. 3 by calculation of the relevant lengths. The drift velocity has already been derived in Eq. (11). Approximating (weak disorder) $\langle 1 / X\rangle=A$, in a time $1 / \omega$, the particle experiences a net average displacement

$$
l_{\text {drift }}=(1-a) / \omega A \text {. }
$$

Likewise, on the same time scale, the particle's root-mean-square diffusive distance is

$$
l_{\text {diff }}=(A \omega)^{-1 / 2} \text {. }
$$

The ratio of the square of the two is

$$
l_{\mathrm{drift}}^{2} / l_{\mathrm{diff}}^{2}=\left[(1-a)^{2} / A^{2} \omega^{2}\right] /[1 / A \omega],
$$

or, more simply $(1-a)^{2} / A \omega$ which, for small $1-a$, is precisely the ratio Eq. (24). Hence, the crossover in frequency behavior can be traced to the same origin as for nonlinear electric field effects as discussed in Ref. 3.

For the first regime (lowest $\omega$ ), one finds

$$
\begin{array}{r}
\sigma_{\text {uncor }}(\omega) \sim a_{0}-a_{1} \omega^{2}+a_{2} i \omega^{3}+\cdots, \\
\omega<(1-a)^{2} / A,
\end{array}
$$

while for the latter regime,

$$
\begin{aligned}
\sigma_{\text {uncor }}(\omega) \sim b_{0}+b_{1}(i \omega)^{1 / 2} & +\cdots, \\
\omega & >(1-a)^{2} / A .
\end{aligned}
$$

The coefficients are positive, and depend on the strength of the disorder through Eq. (23). The limiting behaviors are easily seen if one plots the full solution to Eq. (23). Taking $A=1$ for simplicity, we have calculated the frequency-dependent part of $\sigma(\omega),(\lambda-1)^{2} /\left(\lambda^{2}-a\right)$, against $\omega$, for $a=0.8$ and 0.9. Figure 1 exhibits the real part of this factor for the interesting frequency regime with $a=0.8$. One sees a crossover at $\omega=0.046$ between the twofrequency regimes. The low-frequency driftdominated limiting regime for the frequencydependent part of $\sigma(\omega)$ is exhibited in Fig. 2, and for the imaginary part in Fig. 3. Finally, the higher-frequency diffusion-dominated regimelimiting behavior is exhibited in Fig. 4. Similar plots for $a=0.9$ are exhibited in Figs. 5-7. One sees that crossover takes place at lower frequency, $\omega=0.011$ from Fig. 5 . This follows closely the prediction of Eq. (24) (as it must).

These curves show that the limiting lowfrequency dependence is an immediate and strong function of the nonlinear response. For small elec-

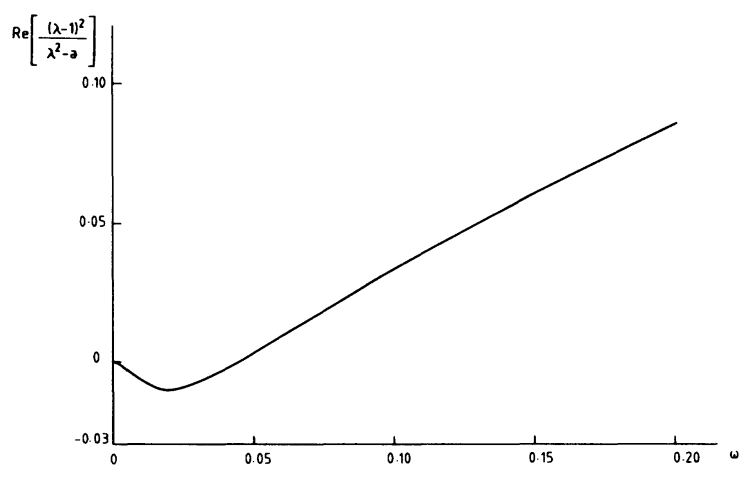

FIG. 1. Plot of the real part of the frequencydependent part of $\sigma(\omega),(\lambda-1)^{2} /\left(\lambda^{2}-a\right)$ (defined in the text), vs $\omega$ for the bias parameter $a=0.8$, and background regular inverse hop rate $A=1$. Note the crossover behavior near $\omega=0.046$. 
tric fields, the drift regime is limited to very small frequencies, increasing in scale as the electric field is enhanced. For the symmetric case, $a=1$, we recover precisely the expression for $\sigma_{\text {uncor }}(\omega)$ given in Refs. 2 and 9, to lowest order in $\omega$.

For correlated $X_{n}$, the argument goes through as before, with the following results for the symmetric case $(a=1)$ :

$$
\begin{aligned}
\sigma_{\text {cor }}(\omega)= & 1 / A+\frac{\left\langle\epsilon_{0}^{2}\right\rangle}{A^{3}}\left(\frac{\lambda-1}{\lambda+1}\right) \\
& +2 \frac{\lambda-1}{\lambda+1} \frac{1}{A^{3}} \sum_{p=1}^{\infty}\left\langle\epsilon_{0} \epsilon_{p}\right\rangle \frac{1}{\lambda^{p}},
\end{aligned}
$$

and the asymmetric case $(a<1)$ :

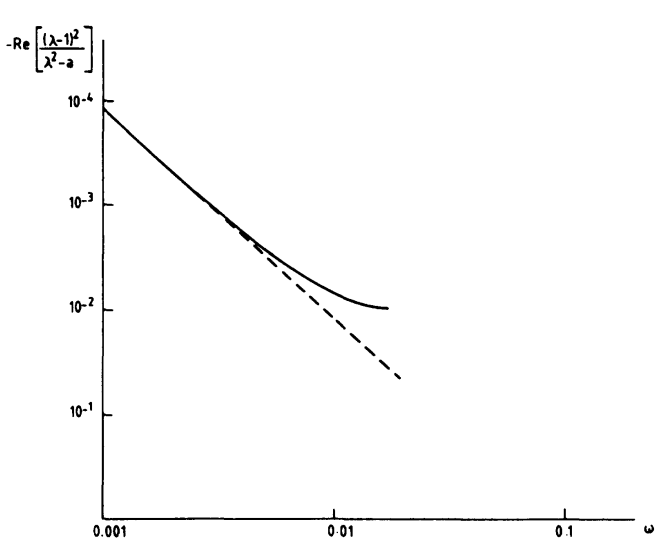

FIG. 2. Same as for Fig. 1, but for small $\omega$ on an expanded scale. The straight dashed line has slope 2, showing that $\sigma_{R}(\omega)$ falls off as $\omega^{2}$ for smallest $\omega$.

$$
\sigma_{\text {cor }}(\omega)=\frac{a}{A}+\frac{\left\langle\epsilon_{0}^{2}\right\rangle}{A^{3}} \frac{a(\lambda-1)^{2}}{\lambda^{2}-a}+\frac{1}{A^{3}} \sum_{p=1}^{\infty}\left\langle\epsilon_{0} \epsilon_{p}\right\rangle\left[\frac{(\lambda-a)^{2}}{\lambda^{2}-a} \frac{a^{p}}{\lambda^{p}}+\frac{a(\lambda-1)^{2}}{\lambda^{2}-a} \frac{1}{\lambda^{p}}-(1-a) a^{p}\right] .
$$

\section{FLUCTUATIONS IN THE AUTOCORRELATION FUNCTION}

One of the more intriguing questions which exact treatments of random one-dimensional systems have been unable to treat is fluctuations about the ensemble average. The simplest quantity, the autocorrelation function, is an example of how difficult such problems are. As discussed in Ref. 1, Sec. X, the variance of the autocorrelation function $P_{0}(t)$, involves the calculation of

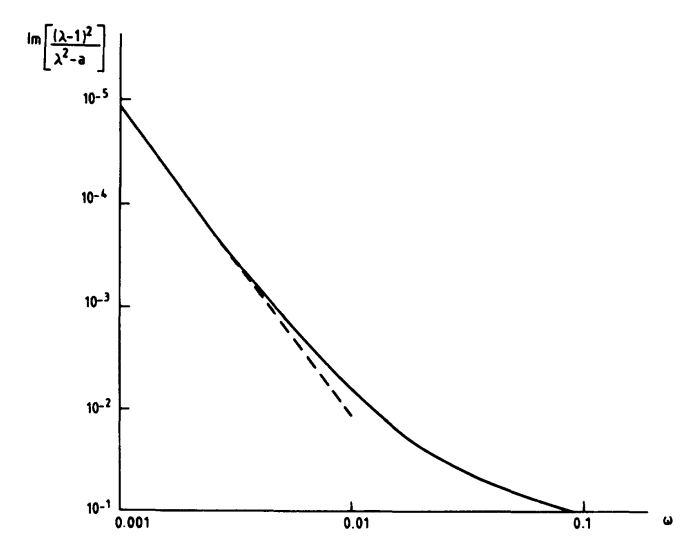

FIG. 3. Same as for Fig. 1, but for the imaginary part of $\sigma(\omega), \sigma_{I}(\omega)$, for small $\omega$ on an expanded scale. The straight dashed line has slope 3 , showing that $\sigma_{I}(\omega)$ increases as $\omega^{3}$ for the smallest $\omega$.

$$
\left\langle P_{0}^{2}(t)\right\rangle=\mathscr{L}^{-1}\left\langle\frac{1}{2 \pi i} \int_{c-i \infty}^{c+i \infty} d \omega^{\prime} \widetilde{P}_{0}\left(\omega^{\prime}\right) \widetilde{P}_{0}\left(\omega-\omega^{\prime}\right)\right\rangle,
$$

where $\widetilde{P}_{0}(\omega)$ is the Laplace transform $(\mathscr{L})$ of $P_{0}(t)$, where

$$
\widetilde{P}_{n}(\omega)=\int_{0}^{\infty} e^{-\omega t} P_{n}(t) d t
$$

Because the $\widetilde{P}_{n}(\omega)$ are correlated ${ }^{1}$ for different $\omega$, the averaging process in Eq. (30) does not commute with the convolution, and one cannot simplify fur-

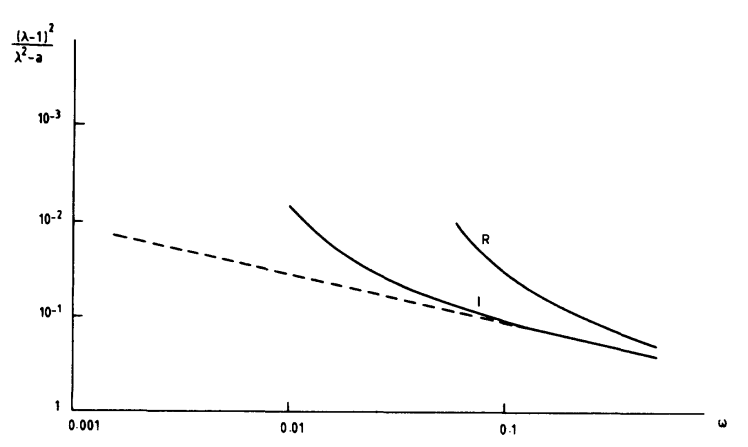

FIG. 4. Same as for Fig. 1, but for the real $(R)$ and imaginary $(I)$ parts of $(\lambda-1)^{2} /\left(\lambda^{2}-a\right)$ above the crossover frequency on an expanded scale. The straight line has slope $\frac{1}{2}$, showing that $\sigma_{I}(\omega)$ rapidly reaches this limiting behavior, but $\sigma_{R}(\omega)$ does so more slowly. 


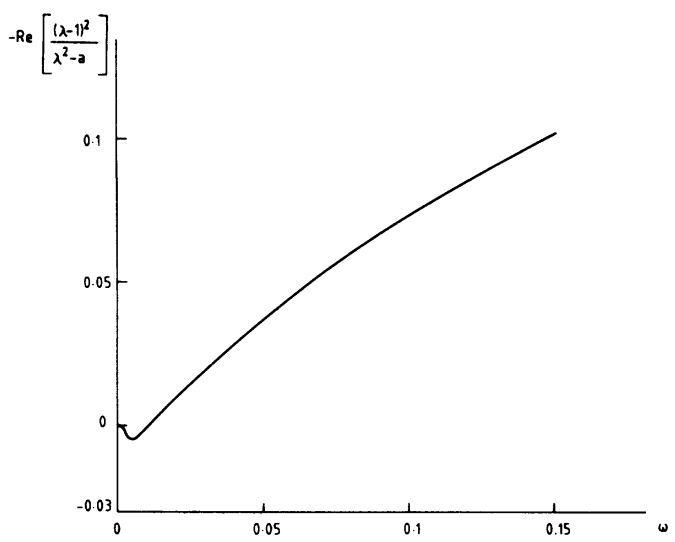

FIG. 5. Plot of the real part of the frequencydependent part of $\sigma(\omega),(\lambda-1)^{2} /\left(\lambda^{2}-a\right)$ (defined in the text), vs $\omega$ for the bias parameter $a=0.9$, and background regular inverse hop rate $A=1$. Note the crossover behavior near $\omega=0.011$.

ther that expression.

Using the approximation of weak disorder, we have been able to make some progress. The Laplace transform of Eq. (1), with the initial condition that the particle is at the origin, $n=0$ at time $t=0$, is

$$
\begin{aligned}
\omega \widetilde{P}_{n}(\omega)= & \delta_{n, 0}+X_{n}\left[a \widetilde{P}_{n+1}(\omega)-\widetilde{P}_{n}(\omega)\right] \\
& +X_{n-1}\left[\widetilde{P}_{n-1}(\omega)-a \widetilde{P}_{n}(\omega)\right] .
\end{aligned}
$$

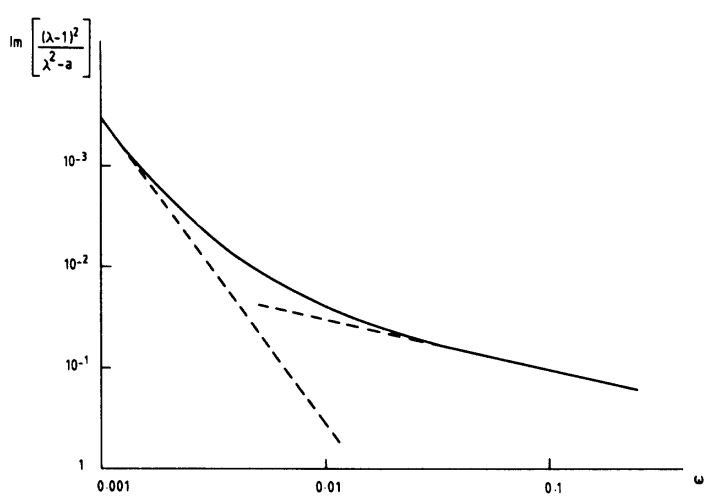

FIG. 6. Same as for Fig. 5, but for $\sigma_{I}(\omega)$ on an expanded scale. The straight dashed lines have slopes 3 and $\frac{1}{2}$, for comparison with limiting behaviors of $\sigma_{I}(\omega)$ for frequencies below and above crossover, respectively.

As before, we expand in the $\epsilon_{n}$ :

$$
\begin{aligned}
\widetilde{P}_{n}(\omega)= & \widetilde{Q}_{n}(\omega)+\sum_{p=-\infty}^{\infty} \alpha_{n, p} \epsilon_{p} \\
& +\sum_{p=-\infty}^{\infty} \sum_{q=-\infty}^{\infty} \beta_{n, p, q} \epsilon_{p} \epsilon_{q}+\ldots,
\end{aligned}
$$

where $\widetilde{Q}_{n}, \alpha_{n, p}$, and $\beta_{n, p, q}$ are all functions of $\omega . \mathrm{A}$ little algebra shows that if the $X_{n}$ are not correlated,

$$
\left\langle\widetilde{P}_{n}(\omega) \widetilde{P}_{n}\left(\omega^{\prime}\right)\right\rangle-\left\langle\widetilde{P}_{n}(\omega)\right\rangle\left\langle\widetilde{P}_{n}\left(\omega^{\prime}\right)\right\rangle=\left\langle\epsilon^{2}\right\rangle \sum_{p=-\infty}^{\infty} \alpha_{n, p}(\omega) \alpha_{n, p}\left(\omega^{\prime}\right),
$$

the $\beta_{n, p, q}$ cancel when taking the difference (33). We need only find the $\alpha_{n, p}(\omega)$ to solve Eq. (33). These are found as before by use of Eq. (32) in Eq. (31). One finds the relations

$$
\omega \widetilde{Q}_{n}=\delta_{n, 0}+(1 / A)\left(a \widetilde{Q}_{n+1}-\widetilde{Q}_{n}+\widetilde{Q}_{n-1}-a \widetilde{Q}_{n}\right)
$$

and

$$
\begin{aligned}
\omega \alpha_{n, p}= & (1 / A)\left[a \alpha_{n+1, p}+\alpha_{n-1, p}-(1+a) \alpha_{n, p}\right] \\
& -\left(1 / A^{2}\right) \delta_{n, p}\left(a \widetilde{Q}_{n+1}-\widetilde{Q}_{n}\right)-\left(1 / A^{2}\right) \delta_{n-1, p}\left(\widetilde{Q}_{n-1}-a \widetilde{Q}_{n}\right) .
\end{aligned}
$$

If $\lambda$ and $\mu$ are the two roots of

$$
a \lambda^{2}-\lambda(1+a+A \omega)+1=0,
$$

with $|\lambda|>|\mu|$, then the $\widetilde{Q}_{n}$ are given by

$$
n \geq 0: \quad \widetilde{Q}_{n}=A \lambda \mu^{n+1} /(\lambda-\mu), \quad n \leq 0: \widetilde{Q}_{n}=A \lambda^{n+1} \mu /(\lambda-\mu) .
$$

The $\alpha_{n, p}$ are likewise given by 


$$
p \geq 0, \quad \alpha_{n, p}=\left\{\begin{array}{l}
-\frac{\lambda \mu}{(\lambda-\mu)^{2}}(\lambda-1)(1-\mu) \mu^{n}, \quad n \geq p+1 \\
\frac{\mu^{2}(\lambda-1)^{2}}{(\lambda-\mu)^{2}} \mu^{p} \lambda^{n-p}, \quad n \leq p
\end{array}\right.
$$

and for

$$
p \leq-1, \quad \alpha_{n, p}=\left\{\begin{array}{l}
\frac{(1-\mu)^{2} \lambda^{2}}{(\lambda-\mu)^{2}} \lambda^{p} \mu^{n-p}, \quad n \geq p+1, \\
-\frac{\lambda \mu}{(\lambda-\mu)^{2}}(\lambda-1)(1-\mu) \lambda^{n}, \quad n \leq p .
\end{array}\right.
$$

Using these expressions, Eq. (33) becomes

$$
\left\langle\widetilde{P}_{0}(\omega) \widetilde{P}_{0}\left(\omega^{\prime}\right)\right\rangle-\left\langle\widetilde{P}_{0}(\omega)\right\rangle\left\langle\widetilde{P}_{0}\left(\omega^{\prime}\right)\right\rangle=\left\langle\epsilon^{2}\right\rangle \frac{\lambda_{1} \lambda_{2} \mu_{1} \mu_{2}\left[\left(\lambda_{1}-1\right)^{2}\left(\lambda_{2}-1\right)^{2} \mu_{1} \mu_{2}+\lambda_{1} \lambda_{2}\left(1-\mu_{1}\right)^{2}\left(1-\mu_{2}\right)^{2}\right]}{\left(\lambda_{1}-\mu_{1}\right)^{2}\left(\lambda_{2}-\mu_{2}\right)^{2}\left(\lambda_{1} \lambda_{2}-\mu_{1} \mu_{2}\right)},
$$

where $\lambda_{1}, \mu_{1}$ and $\lambda_{2}, \mu_{2}$ are the roots of Eq. (35) (remembering $|\lambda|>|\mu|$ ) for $\omega$ and $\omega^{\prime}$, respectively. For the symmetric case, $a=1$ and Eq. (36) "simplifies" to $\left(\mu_{1}=1 / \lambda_{1}, \mu_{2}=1 / \lambda_{2}\right)$,

$$
\left\langle\widetilde{P}_{0}(\omega) \widetilde{P}_{0}\left(\omega^{\prime}\right)\right\rangle-\left\langle\widetilde{P}_{0}(\omega)\right\rangle\left\langle\widetilde{P}_{0}\left(\omega^{\prime}\right)\right\rangle=\left\langle\epsilon^{2}\right\rangle \frac{2 \lambda_{1}^{2} \lambda_{2}^{2}}{\left(\lambda_{1}+1\right)^{2}\left(\lambda_{2}+1\right)^{2}\left(\lambda_{1}^{2} \lambda_{2}^{2}-1\right)} .
$$

We wish the inverse Laplace transform of this expression, a formidable task which so far has eluded us for general $\omega, \omega^{\prime}$. However, for small $\omega, \omega^{\prime}$, $\lambda=1+(A \omega)^{1 / 2}$, whence Eq. (37) reduces to

$$
\begin{aligned}
\left\langle\widetilde{P}_{0}(\omega) \widetilde{P}_{0}\left(\omega^{\prime}\right)\right\rangle-\left\langle\widetilde{P}_{0}(\omega)\right\rangle\left\langle\widetilde{P}_{0}\left(\omega^{\prime}\right)\right\rangle & =\frac{\left\langle\epsilon^{2}\right\rangle}{16 \sqrt{A}\left(\sqrt{\omega}+\sqrt{\omega^{\prime}}\right)} .
\end{aligned}
$$

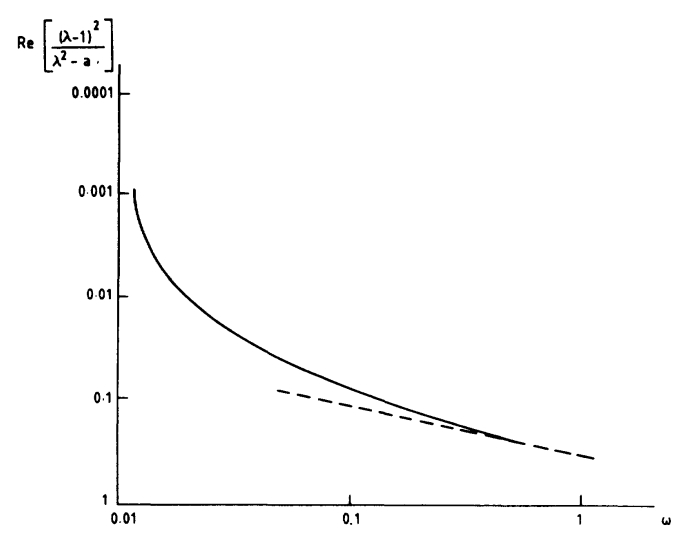

FIG. 7. Same as for Fig. 5, but for the real part of $(\lambda-1)^{2} /\left(\lambda^{2}-a\right)$ above the crossover frequency on an expanded scale. The straight dashed line has slope $\frac{1}{2}$ for comparison with the predicted limiting behavior.
The inverse Laplace transform of $\left(\sqrt{\omega}+\sqrt{\omega^{\prime}}\right)^{-1}$ is known, so that the long-time behavior of the variance of $P_{0}(t)$ is approximately given by

$$
\begin{array}{r}
\left\langle P_{0}^{2}(t)\right\rangle-\left\langle P_{0}(t)\right\rangle^{2}=\frac{\left\langle\epsilon^{2}\right\rangle}{64 \sqrt{2 \pi} \sqrt{A}} t^{-3 / 2}, \\
t \rightarrow \infty .
\end{array}
$$

Comparing with $\left\langle P_{0}(t)\right\rangle=\sqrt{A} /\left(2 \sqrt{\pi} t^{1 / 2}\right)$, we find

$$
\frac{\left\langle P_{0}^{2}(t)\right\rangle-\left\langle P_{0}(t)\right\rangle^{2}}{\left\langle P_{0}(t)\right\rangle^{2}}=\frac{\sqrt{\pi}}{16 \sqrt{2}} \frac{\left\langle\epsilon^{2}\right\rangle}{A^{2}}\left(\frac{A}{t}\right]^{1 / 2},
$$

This is a highly satisfying result, for it demonstrates that the relative variance of $P_{0}(t)$ falls off with increasing time as $t^{-1 / 2}$ for the symmetric case with weak disorder. The asymmetric case is more complicated, but it is at least formulated [Eq. (36)] in terms of the Laplace transformed quantities. The full problem, for arbitrary disorder, seems out of reach at present.

\section{SUMMARY}

We have considered the random one-dimensional near-neighbor hopping transport problem for asymmetric hopping rates with constant bias. This would correspond to the problem of measurement of the frequency-dependent conductivity in the presence of 
a large dc electric field. We have derived expressions for the drift velocity and $\sigma(\omega)$ and have evaluated them

(a) exactly for the drift velocity contribution to the dc conductivity,

(b) exactly in the high-frequency limit, and

(c) exactly for weak disorder.

In case (c), we have shown that a crossover frequency exists, proportional to the bias, (i.e., strength of the electric field) below which the real part of the conductivity diminishes as $\omega^{2}$ and the imaginary part increases as $\omega^{3}$. Above the crossover frequency, the real and imaginary parts increase as $\omega^{1 / 2}$. We have exhibited numerous graphs which show the real and imaginary parts of $\sigma(\omega)$ over the applicable frequency range.

We have limited ourselves to the first correction term for the $R_{n}$ [Eq. (18)] when we work to lowest order in the disorder [case (c) above]. To that order, the frequency dependence we have found for $\sigma(\omega)$ is exact. One can pose the question of the effect on $\sigma(\omega)$ of introducing higher-order corrections in Eq. (18). Such a task is formidable, but we know the answer in the symmetric case $(a=1)$. There, the $\sigma(\omega)$ can be expanded as a power series in $\left\langle\epsilon^{n}\right\rangle$. One finds ${ }^{9}$ that these terms carry with them only higher powers of $\omega^{1 / 2}$ :

$$
\sigma(\omega) \propto \sigma(0)+O\left(\left\langle\epsilon^{2}\right\rangle\right) \omega^{1 / 2}+O\left(\left\langle\epsilon^{3}\right\rangle\right) \omega+\cdots .
$$

Thus, to lowest order in $\left\langle\epsilon^{2}\right\rangle$, one obtains the first frequency-dependent correction to $\sigma(\omega)$, proportional to $\omega^{1 / 2}$. To higher order in $\epsilon$, one obtains yet higher powers of $\omega^{1 / 2}$. This suggests (but we have no proof) that the next term in an expansion in the disorder for the asymmetric case would yield frequency corrections of a higher power than we have found in Sec. IV. Said another way, it may be the case that the weak-disorder limit may in fact generate only the lowest-frequency correction to the dc conductivity in the asymmetric limit (as it does in the symmetric case), with higher-frequency corrections arising from higher-order terms in the expansion in powers of the disorder (as it does in the symmetric case). This conjecture remains to be proven, but if true it means that our expressions for case (c) above can be regarded as a low-frequency expansion for the situation where the disorder is not necessarily weak.

Finally, we have shown for weak disorder that one can obtain a closed form expression for the Laplace transform of the variance of the autocorrelation function. We have succeeded in obtaining the inverse for the symmetric case, and have shown that fluctuations above the mean value fall off as $t^{-1 / 2}$ for long times.

\section{ACKNOWLEDGMENTS}

The authors wish to acknowledge very helpful discussions with Dr. J. Bernasconi and Dr. W. R. Schneider, and receipt of their interesting work on transport for asymmetric hopping rates prior to publication. The research of one of us (R.O.) was supported in part by the U.S. National Science Foundation and the U.S. Office of Naval Research.
*Permanent address: Department of Physics, University of California, Los Angeles, California 90024.

1S. Alexander, J. Bernasconi, W. R. Schneider, and R. Orbach, Rev. Mod. Phys. 53, 175 (1981).

2S. Alexander and R. Orbach, Physica 107B, 675 (1981).

${ }^{3}$ S. Alexander, J. Bernasconi, W. R. Schneider, and R. Orbach, in Physics in One Dimension, Vol. 23 of Springer Series in Solid State Sciences, edited by J. Bernasconi and T. Schneider (Springer, Berlin, 1981), p. 277.

${ }^{4}$ S. Alexander, J. Bernasconi, W. R. Schneider, R. Biller, W. G. Clark, G. Gruner, R. Orbach, and A. Zettl. Phys. Rev. B 24, 7474 (1981); S. Alexander, J. Bernasconi, W. R. Schneider, R. Biller, and R. Orbach,
Mol. Cryst. Liq. Cryst. $\underline{85}, 121$ (1982).

${ }^{5}$ M. J. Stephen, J. Phys. C 14, L1077 (1981).

${ }^{6}$ B. Derrida and Y. Pomeau, Phys. Rev. Lett. 48, 627 (1982).

7J. Bernasconi and W. R. Schneider, J. Phys. A 15, L729 (1982).

${ }^{8}$ M. J. Rice, S. Strassler, and W. R. Schneider, in One Dimensional Conductors, edited by H. G. Schuster (Springer, Berlin, 1975), pp. 307-309.

${ }^{9}$ Reference 2, and J. Machta, Phys. Rev. B 24, 5260 (1981); R. Zwanzig, J. Stat. Phys. 28, 127 (1982); I. Webman and J. Klafter, Phys. Rev. B 26, 5950 (1982). 